\title{
Watering Eyes
}

National Cancer Institute

\section{Source}

National Cancer Institute. Watering Eyes. NCI Thesaurus. Code C78717.

Tearing eyes; a condition caused either by tear overproduction or impaired drainage of the tear duct. Causes of tear overproduction include eye irritation (e.g., eye infection, foreign body in the eye), headache, pain reaction, allergies, sinusitis, and injury to the eye. 